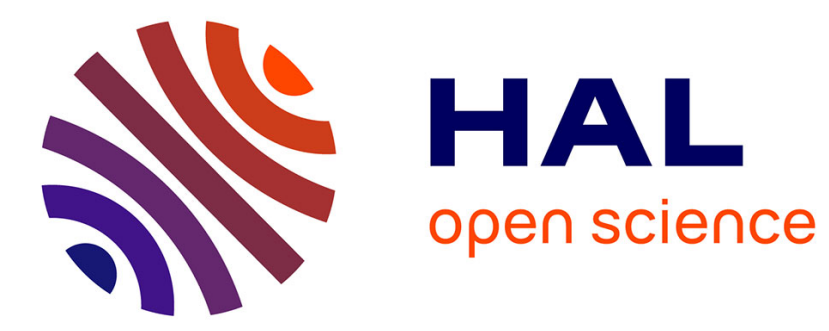

\title{
L1 Adaptive Control of Parallel Kinematic Manipulators: Design and Real-Time Experiments
}

\author{
Moussab Bennehar, Ahmed Chemori, François Pierrot
}

\section{To cite this version:}

Moussab Bennehar, Ahmed Chemori, François Pierrot. L1 Adaptive Control of Parallel Kinematic Manipulators: Design and Real-Time Experiments. ICRA: International Conference on Robotics and Automation, May 2015, Seattle, WA, United States. pp.1587-1592, 10.1109/ICRA.2015.7139400 . hal-01176534

\section{HAL Id: hal-01176534 \\ https://hal.science/hal-01176534}

Submitted on 15 Jul 2015

HAL is a multi-disciplinary open access archive for the deposit and dissemination of scientific research documents, whether they are published or not. The documents may come from teaching and research institutions in France or abroad, or from public or private research centers.
L'archive ouverte pluridisciplinaire HAL, est destinée au dépôt et à la diffusion de documents scientifiques de niveau recherche, publiés ou non, émanant des établissements d'enseignement et de recherche français ou étrangers, des laboratoires publics ou privés. 


\title{
$\mathcal{L}_{1}$ Adaptive Control of Parallel Kinematic Manipulators: Design and Real-Time Experiments
}

\author{
Moussab Bennehar ${ }^{1}$, Ahmed Chemori ${ }^{1}$ and François Pierrot ${ }^{1}$
}

\begin{abstract}
In this paper, the recently developed $\mathcal{L}_{1}$ adaptive control strategy is experimentally validated for the first time on a parallel kinematic manipulator. The $\mathcal{L}_{1}$ adaptive controller is known for its decoupled estimation and control loops which enables fast adaptation while guaranteeing robustness of the closed-loop system. The control scheme is experimentally implemented on a 4-DOFs parallel kinematic manipulator. Based on the obtained experimental results, a comparative study shows that the proposed $\mathcal{L}_{1}$ adaptive controller outperforms the PD controller in terms of tracking performance thanks to the compensation of the nonlinearities in the adaptive controller.
\end{abstract}

\section{INTRODUCTION}

Control of Parallel Kinematic Manipulators (PKMs) has always been described as a challenging task in nonlinear control community. Indeed, the interconnected structure of rigid bodies in PKMs gives rise to complex nonlinearities that have to be carefully taken into consideration in the control scheme [1]. The earlier control schemes implemented on mechanical manipulators were conventional single-axis controllers such as the PD [2] and its nonlinear counterpart (NPD) [3]. Singleaxis controllers are the most adopted schemes in industrial world thanks to their simplicity and efficiency. However, they result in a poor tracking performance especially on high acceleration tasks. Fixed model-based controllers (e.g Computed Torque [4], PD+ [5], Augmented PD [6], ...) were consequently introduced to overcome the limitations of single-axis controllers and particularly, to improve the tracking performance. In this class of controllers, a nonlinear loop based on the dynamics of the manipulator is included in order to compensate for the inherent nonlinearities. Nevertheless, they require an accurate dynamic model of the manipulator and its parameters to be able to adequately cancel the nonlinearities. For this reason, fixed model-based controllers are not suitable for real industrial applications where the dynamic model is often time-varying or unknown (e.g varying payload).

In order to overcome such limitations of fixed modelbased controllers, adaptive control theory seemed to be a suitable solution and therefore, was introduced to mechanical manipulators [7]. Similar to fixed model-based controllers, the control architecture features a nonlinear loop to cancel the inherent nonlinearities. Besides, the control architecture is appended with an estimation loop that adjusts the varying parameters of the controller in real-time according to the

\footnotetext{
${ }^{1}$ M. Bennehar, A. Chemori and F. Pierrot are with the Robotics Department of Laboratoire d'Informatique, de Robotique et de Microélectonique de Montpellier (LIRMM) 161 rue Ada, 34090 Montpellier, France. Email: \{bennehar, chemori, pierrot\}alirmm.fr
}

variations of the system. Although adaptive control was widely implemented on serial manipulators [8], [9], [10], it has not gained the same success with PKMs, despite the fact that they share similar properties [11]. Since this paper is focused on PKMs, we limit our discussion in the sequel to adaptive controllers applied to PKMs.

In [12], the Desired Compensation Adaptive Law (DCAL) has been used to control the Hexaglide, a 6-DOFs PKM intended to be used for high speed milling. The DCAL controller allowed to estimate both inertial and friction parameters of the Hexaglide in real-time. DCAL was recently extended in [13] by replacing the fixed gains in the feedback loop with nonlinear varying ones. The superiority of the proposed extended DCAL controller in terms of tracking performance was demonstrated through real-time experiments on a 4-DOF Redundantly Actuated PKM (RA-PKM). In [14], a task-space adaptive controller was proposed to control a 2-DOFs RA-PKM. The gradient descent algorithm has been used to minimize a tracking performance index. The stability analysis of the proposed controller has been conducted based on the Lyapunov theory and real-time experiments showed its relevance. In [15], the Backstepping algorithm has been employed to design an adaptive controller for the set-point control of a 2-DOFs planar PKM. Although the aforementioned adaptive controllers may seem quite different, they share one particular limitation. Indeed, in order to achieve fast convergence of the parameters and hence, a good tracking performance, the adaptation gain has to be increased. However, increasing the adaptation gain may lead to parameter divergence and risk of instability.

Recently, a new control scheme called $\mathcal{L}_{1}$ adaptive control has been developed to overcome the aforementioned shortcoming and release such a compromise [16], [17]. Indeed, the $\mathcal{L}_{1}$ adaptive control essentially aims at decoupling robustness and adaptation through introducing a low-pass filter into the control architecture. In contrast with Model Reference Adaptive Control (MRAC), the $\mathcal{L}_{1}$ adaptive control strategy focuses on the feasibility of the controller by partial compensation of the uncertainties lying inside the control channel bandwidth [18]. The first validations of $\mathcal{L}_{1}$ adaptive control were through numerical simulations on aerial vehicles [19]. Then, it started gaining an increased interest and was successfully implemented on various systems such as underwater vehicles [20], serial mechanical manipulators [21], underactuated mechanical systems [22], and others.

The main contribution of this paper is to experimentally validate the $\mathcal{L}_{1}$ adaptive control theory on a more complex, highly nonlinear system; namely the parallel manipulator 
VELOCE. The remainder of this paper is organized as follows. In Section II, a brief description of the modeling of VELOCE PKM is provided. Section III is dedicated to the problem formulation of the $\mathcal{L}_{1}$ adaptive control and its application to PKMs. The prototype testbed as well as real-time results are presented and discussed in Section IV. Finally, conclusions and future work are given in section V.

\section{DESCRIPTION AND DYNAMIC MODELING OF VELOCE: A 4-DOF FULLY PKM}

VELOCE is a 4-DOFs fully PKM, which means that it has the same number of kinematic chains as DOFs [1]. Each kinematic chain is a serial arrangement of an actuator (i.e. its stator part), an arm (including the rotor part of the actuator) and a forearm. Its platform (called a traveling plate) is capable of performing three translations $(x, y, z)$ along the $\mathrm{X}-\mathrm{Y}-\mathrm{Z}$ axes and one rotation $(\alpha)$ about the $\mathrm{Z}$ axis. Fig. 1 illustrates a schematic view of the CAD design of VELOCE as well as its workspace. In the sequel, a brief description of the dynamic model of VELOCE, necessary for the subsequent theoretical development, is provided.

For the dynamic modeling of VELOCE, the following assumptions, commonly used in Delta-like robots, have been considered [23]:

- Both dry and viscous frictions of all joints are neglected since the joints are carefully designed such that the friction effects are minimized.

- The inertia of the forearms is neglected and their mass is split up into two equivalent parts, one is added to the mass of the arm while the other one is considered with the traveling plate.

- Gravity is not considered since we are dealing with high acceleration motions.

The dynamic parameters of the robot are summarized in Table I and its dynamic model is established through analyzing the equilibrium of the arms and the traveling plate separately. Then, the complete dynamic model is obtained by summing-up these two quantities.

\section{A. Equilibrium of the arms}

The relationship between the torque vector $\Gamma \in \mathbb{R}^{4}$ and the joint accelerations vector $\ddot{q} \in \mathbb{R}^{4}$ is formulated as

$$
\Gamma-J_{q}^{T} F=\left(I_{\text {act }}+I_{\text {arm }}+\frac{l^{2} M_{\text {forearm }}}{2}\right) \ddot{q}
$$

where $I_{\text {act }} \in \mathbb{R}^{4 \times 4}$ and $I_{\text {arm }} \in \mathbb{R}^{4 \times 4}$ are the inertia matrices of the motor drives and the arms, respectively. $M_{\text {forearm }} \in$ $\mathbb{R}^{4 \times 4}$ is the mass matrix of the forearms, $J_{q} \in \mathbb{R}^{4 \times 4}$ is the joint Jacobian matrix, $F \in \mathbb{R}^{4}$ is the force vector associated to the acceleration of the traveling plate. $l=\operatorname{diag}\left(l_{1}, \ldots, l_{4}\right)$ with $l_{i}$ the length of the $i$ th arm, $\Gamma \in \mathbb{R}^{4}$ is the control input vector.

\section{B. Equilibrium of the traveling plate}

The equation of motion of the traveling plate is

$$
J_{x}^{T} F=\left(M_{t p}+4 \frac{M_{\text {forearm }}}{2}\right) \ddot{X}
$$

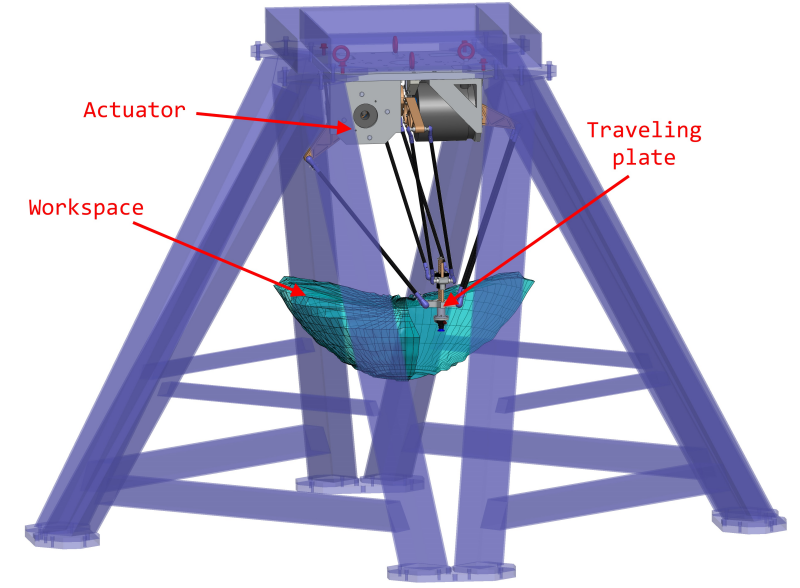

Fig. 1. CAD schematic view of VELOCE PKM

where $J_{x} \in \mathbb{R}^{4 \times 4}$ is the Cartesian Jacobian matrix, $M_{t p} \in$ $\mathbb{R}^{4 \times 4}$ is the mass matrix of the traveling plate including eventual payload and $\ddot{X} \in \mathbb{R}^{4}$ is its acceleration vector. It is worth noting that the complete Jacobian matrix of the manipulator $J_{m}(q) \in \mathbb{R}^{4 \times 4}$, which is a function of the manipulator's pose, is given by $J_{m}=J_{q}^{-1} J_{x}$.

\section{Complete equilibrium and full dynamic model}

The complete equilibrium of the manipulator is obtained by substituting (1) in (2) as follows

$$
\begin{aligned}
\ddot{X}= & \left(M_{t p}+4 \frac{M_{\text {forearm }}}{2}\right)^{-1} J_{m}^{T}[\Gamma \\
& \left.-\left(I_{\text {act }}+I_{\text {arm }}+\frac{l^{2} M_{\text {forearm }}}{2}\right) \ddot{q}\right]
\end{aligned}
$$

The simplified dynamic model (3) is essential in any model-based control scheme. It is important to note that (3) can be expressed using only Cartesian quantities by using the adequate kinematic relationships that map the joint and the Cartesian spaces. To sum up, the dynamic midel of VELOCE can be finally expressed in Cartesian space as follows

$$
\ddot{X}=\left(M_{t o t}+J_{m}^{T} I_{t o t} J_{m}\right)^{-1} J_{m}^{T}\left(\Gamma-I_{t o t} \dot{J}_{m} \dot{X}\right)
$$

where $M_{t o t}=M_{t p}+4 \frac{M_{\text {forearm }}}{2}$ is the total mass matrix of the manipulator and $I_{\text {tot }}=I_{\text {act }}+I_{\text {arm }}+\frac{l^{2} M_{\text {forearm }}}{2}$ is its total inertia matrix. Similarly, using the appropriate kinematic relationships, always valid away from singularities, the dynamic model of VELOCE can be written in the standard joint space form as

$$
M(q) \ddot{q}+N(q, \dot{q})=\Gamma(t)
$$

TABLE I

DYNAMIC PARAMETERS OF VELOCE

\begin{tabular}{|c|c|c|c|}
\hline$M_{t p}[\mathrm{Kg}]$ & $M_{\text {forearm }}[\mathrm{Kg}]$ & $I_{a c t}\left[\mathrm{Kg} \cdot \mathrm{m}^{2}\right]$ & $I_{\text {arm }}\left[\mathrm{Kg} \cdot \mathrm{m}^{2}\right]$ \\
\hline 0.257 & 0.080 & 0.0041 & 0.0053 \\
\hline
\end{tabular}


where $M(q)=I_{t o t}+\left(J_{m}^{T}\right)^{-1} M_{t o t}\left(J_{m}^{T}\right)^{-1}$ is the inertia matrix and $N(q, \dot{q})=\left(J_{m}^{T}\right)^{-1} M_{t o t}\left(J_{m}^{T}\right)^{-1} \dot{J}_{m} J_{m}$ is the Coriolis and centrifugal forces vector. It is worth noting that the structure of the dynamic quantities in this representation is very complex and highly nonlinear due to the inherent complex nonlinearities in the Jacobian matrices. The formulation (4) is more suitable for joint space control design and will then be used in the subsequent development.

\section{PROPOSED CONTROL SOLUTION: $\mathcal{L}_{1}$ ADAPTIVE CONTROL}

Let $q_{d}(t) \in \mathbb{R}^{4}$ be the desired joint position vecotor and $\dot{q}_{d}(t) \in \mathbb{R}^{4}$ the corresponding desired velocity vector. The desired joint trajectories (i.e positions and velocities) are issued from a trajectory generation process which computes in real-time the joint quantities corresponding to a certain task defined in Cartesian space. Consider the combined tracking error $r(t) \in \mathbb{R}^{4}$ as follows

$$
r=\left(\dot{q}-\dot{q}_{d}\right)+\Lambda\left(q-q_{d}\right)
$$

where $\Lambda \in \mathbb{R}^{4 \times 4}$ is a symmetric positive-definite matrix. The control input vector $\tau(t)$ is the combination of two separate terms, a fixed state-feedback term characterizing the evolution of the transient response of the system and an adaptive term that compensates for the nonlinearities of the system, that is

$$
\tau(t)=A_{m} r(t)+\tau_{a d}(t)
$$

where $A_{m} \in \mathbb{R}^{4 \times 4}$ is a Hurwitz matrix characterizing the transient response of the system and $\tau_{a d}(t) \in \mathbb{R}^{4}$ is the adaptive component to be designed later. The time derivative of (6) combined with (7) and (5) leads to the following error dynamics

$$
\dot{r}(t)=A_{m} r(t)+\tau_{a d}(t)-\eta(t, \zeta(t)), \quad r(0)=r_{0}
$$

where $\zeta=[r, q]^{T}$ and $\eta(t, \zeta(t))$ is a nonlinear function that gathers all the nonlinearities of the system including eventual external disturbances. The function $\eta(t, \zeta(t))$ can be parametrized, under some reasonable assumptions, using the truncated infinity norm of $r(t)$ as regressor as follows [18]

$$
\eta(t, \zeta(t))=\theta(t)\left\|r_{\tau}\right\|_{\mathcal{L}_{\infty}}+\sigma(t), \quad \forall t \in[0, \tau]
$$

where $\theta(t), \sigma(t) \in \mathbb{R}^{4}$ are differentiable functions and $\left\|(.)_{\tau}\right\|_{\mathcal{L}_{\infty}}$ denotes the truncated $\mathcal{L}_{\infty}$-norm of $(.) . r_{\tau}(t)$ is the truncation of $\mathrm{r}(\mathrm{t})$ defined by

$$
r_{\tau}(t)=\left\{\begin{aligned}
r(t), & 0 \leq t \leq \tau \\
0, & t>\tau
\end{aligned}\right.
$$

From this parametrization, it follows that (8) can be rewritten as:

$\dot{r}(t)=A_{m} r(t)+\tau_{a d}(t)-\left(\theta(t)\left\|r_{t}\right\|_{\mathcal{L}_{\infty}}+\sigma(t)\right), \quad r(0)=r_{0}$

If all the nonlinearities of the system are perfectly known (i.e perfect dynamic model, ideal working conditions, no external disturbances, etc.), putting $\tau_{a d}(t)=\eta(t, \zeta(t))$ in
(8) yields to an exponentially stable system $\dot{r}(t)=A_{m} r(t)$. Which means that $r(t)$ will converge to zero as fast as specified by the choice of $A_{m}$; consequently $q$ will approach $q_{d}$ since (6) is a stable system. However, it is practically impossible to know perfectly all the nonlinearities of the system. Then, the adaptive control term $\tau_{a d}(t)$ can be designed in order to cancel the nonlinearity $\eta(t, \zeta(t))$. Since this last one is not well known, the objective of the adaptive control term $\tau_{a d}(t)$ would be to estimate and compensate $\eta(t, \zeta(t))$. For that, consider the following state predictor

$$
\begin{aligned}
\dot{\hat{r}}(t)= & A_{m} \hat{r}(t)+\tau_{a d}(t)-\left(\hat{\theta}(t)\left\|r_{t}\right\|_{\mathcal{L}_{\infty}}+\hat{\sigma}(t)\right) \\
& -K \tilde{r}(t), \quad \hat{r}(0)=r_{0}
\end{aligned}
$$

where $\tilde{r}(t) \triangleq \hat{r}(t)-r(t)$ is the prediction error and $K \in \mathbb{R}^{4 \times 4}$ is a design parameter to be tuned to reject high-frequency noise [21]. $\hat{\theta}(t)$ and $\hat{\sigma}(t)$ are estimates of $\theta(t)$ and $\sigma(t)$ respectively, obtained through the following projection-based adaptation laws [18] in order to ensure the boundedness of their infinity norms

$$
\begin{aligned}
& \dot{\hat{\theta}}(t)=\Gamma \operatorname{Proj}\left(\hat{\theta}(t), P \tilde{r}(t)\left\|r_{t}\right\|_{\mathcal{L}_{\infty}}\right), \quad \hat{\theta}(0)=\hat{\theta}_{0} \\
& \dot{\hat{\sigma}}(t)=\Gamma \operatorname{Proj}(\hat{\sigma}(t), P \tilde{r}(t)), \quad \hat{\sigma}(0)=\hat{\sigma}_{0}
\end{aligned}
$$

where $\Gamma \in \mathbb{R}^{+}$is the adaptive gain, $P=P^{T}>0$ is the solution to the algebraic Lyapunov equation $A_{m}^{T} P+$ $P A_{m}=-Q$ for some arbitrary matrix $Q=Q^{T}>0$. The projection operator $\operatorname{Proj}(\theta, y)$ does not modify the value of $y$ if $\theta$ belongs to a certain sphere defined with a maximum accepted value $\theta_{\max }$, it only modifies $y$ when $\theta$ takes values outside the allowed range. Consequently the estimated parameters always satisfy the constraints $\|\hat{\theta}\|_{\infty}<$ $\theta_{b}, \quad\|\hat{\sigma}\|_{\infty}<\sigma_{b}, \quad \forall t \in[0, T]$. The adaptive control term $\tau_{a d}(t)$ is the output of the following system

$$
\tau_{a d}(s)=C(s) \hat{\eta}(s)
$$

where $\hat{\eta}(s)$ is the Laplace transform of $\hat{\eta}(t)=$ $\left(\hat{\theta}(t)\left\|r_{t}\right\|_{\mathcal{L}_{\infty}}+\hat{\sigma}(t)\right)$ and $C(s)$ is a diagonal matrix whose elements are BIBO-stable strictly proper transfer functions satisfying a unity DC gain and zero initialization for their state-space realizations.

The objective of the proposed controller is to drive the tracking error $r$ to its desired value $r_{d}=0$ while ensuring that all signals remain bounded and that the control signal does not exceed the control channel bandwidth [18]. Consequently, the $\mathcal{L}_{1}$ adaptive controller aims at partial compensation of the nonlinearities in order to maintain the robustness of the system. Therefore, the adaptive gain $\Gamma$ can be increased without altering the robustness of the closedloop system. One major advantage of this control scheme lies in the fact that it is a model-free adaptive control. Indeed, in contrast with the widely developed model-based adaptive controllers for mechanical manipulators, the control law in (7) does not require any knowledge about the dynamics of the system.

To sum up, the overall block diagram of the proposed $\mathcal{L}_{1}$ adaptive controller is shown in Fig. 2. 


\section{EXPERIMENTAL RESULTS}

\section{A. Experimental testbed of VELOCE PKM}

In order to validate the proposed adaptive controller, real-time experiments were conducted on the experimental testbed of the prototype of VELOCE PKM, shown in Fig. 3 . The actuators of the robot are the TMB0140-100-3RBS ETEL direct-drive motors. They can provide a maximum peak torque of $127 \mathrm{Nm}$ and they are able to reach $550 \mathrm{rpm}$ of maximum speed. Each actuator is equipped with a contactfree incremental optical encoder providing a total number of 5000 pulses per revolution. The assembled manipulator is capable of reaching $10 \mathrm{~m} / \mathrm{s}$ of maximum linear velocity of the traveling plate, $200 \mathrm{~m} / \mathrm{s}^{2}$ of maximum acceleration and is able to carry a maximum payload of $10 \mathrm{Kg}$. The control architecture is implemented using Matlab/Simulink from Mathworks and compiled using the XPC Target realtime toolbox. Then, the resulting low-level code is uploaded to the target PC, an industrial computer executing the controlloop at a frequency of $10 \mathrm{KHz}$ (i.e. a sample time of $0.1 \mathrm{~ms}$ ).

\section{B. Real-time experiments}

In order to highlight the outcomes of the proposed $\mathcal{L}_{1}$ adaptive controller, its performance is proposed to be compared with the one of a PD controller. The PD controller in joint space can be expressed as [2]

$$
\tau_{P D}(t)=K_{p}\left(q_{d}(t)-q(t)\right)+K_{d}\left(\dot{q}_{d}(t)-\dot{q}(t)\right)
$$

where $K_{p}, K_{d} \in \mathbb{R}^{4 \times 4}$ are positive definite feedback gains usually taken as diagonal matrices (i.e no coupling between the joints is considered). The PD feedback gains providing the best results were tuned by trial and error leading to the following values: $K_{p}=10^{2} \times \operatorname{diag}(39,39,39,39), K_{d}=$ $\operatorname{diag}(6,6,6,6)$. The parameters of the $\mathcal{L}_{1}$ adaptive controller providing the best results are summarized in Table II.

The reference trajectories were generated in Cartesian space using $5^{\text {th }}$ order polynomials. The traveling plate of the manipulator has to follow several point-to-point trajectories within the workspace (including some rotations as well) away from singularities. The duration of each point-to-point trajectory is fixed to 0.15 seconds. The desired joint trajectories are then computed by solving the Inverse Kinematics problem in real-time while the actual joint velocities were

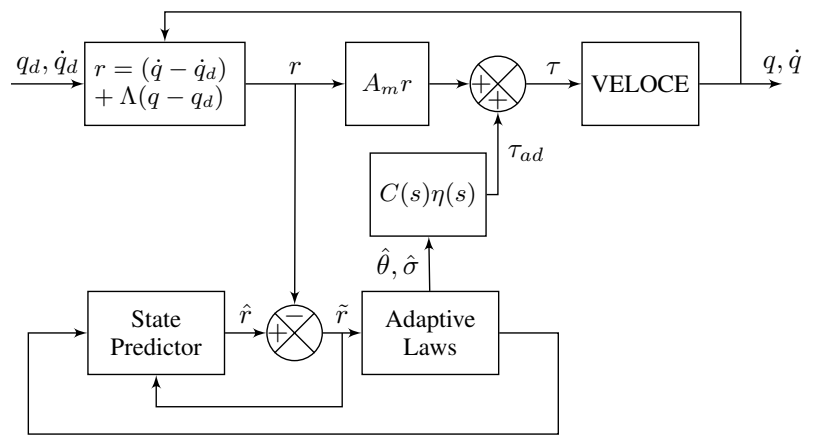

Fig. 2. Block diagram of the $\mathcal{L}_{1}$ adaptive controller

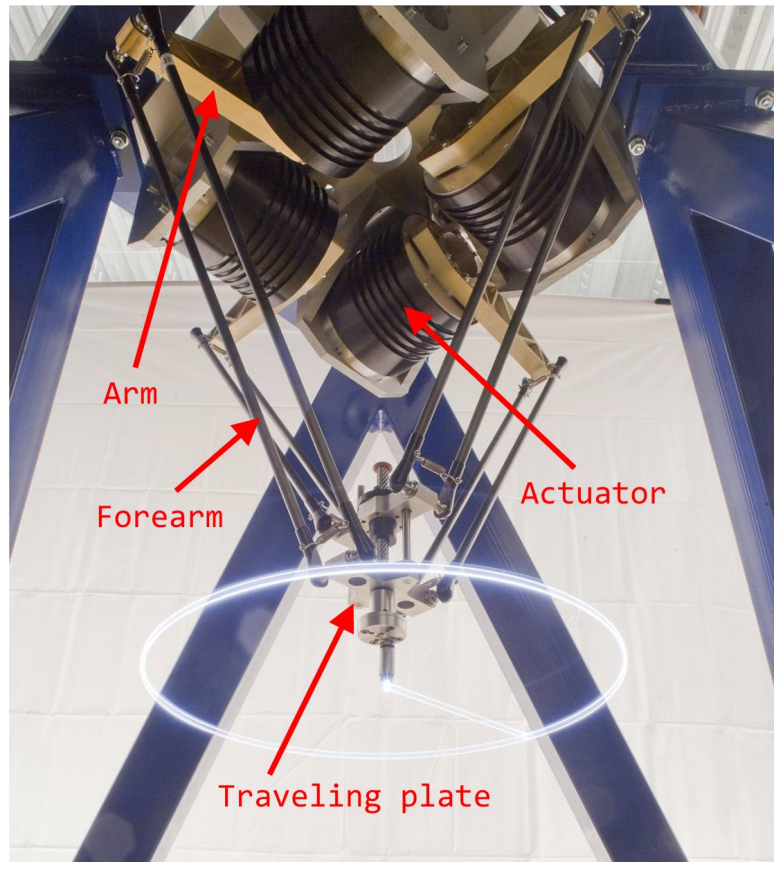

Fig. 3. View of the experimental setup of VELOCE PKM

obtained using a numerical differentiation of the encoders' position measurements. The measured Cartesian positions are computed by solving the Forward Kinematics problem using the measured joint positions.

A comparison between the PD and the $\mathcal{L}_{1}$ adaptive controllers in terms of joint tracking errors is illustrated in Fig. 4. For the seek of clarity, the plots are zoomed around the interval $[8,10]$ seconds. It can be clearly seen that the tracking errors in the case of PD are greater than those of the $\mathcal{L}_{1}$ adaptive controller. The proposed controller significantly improves the tracking capabilities of the manipulator thanks to the compensated nonlinearities.

Similarly, a comparison between both controllers in terms of Cartesian tracking performance is shown in Fig. 5. Once again, the $\mathcal{L}_{1}$ adaptive controller outperforms the PD controller in all axes. The improvement is more noticeable on the $\mathrm{x}$ and $\mathrm{y}$ axes.

To quantify the enhancement brought by the $\mathcal{L}_{1}$ adaptive controller, we evaluate the tracking errors according to the following RMS-based (RMS: Root Mean Square) criteria where the translational movements are separated from the

TABLE II

SUMMARY OF THE PARAMETERS OF THE $\mathcal{L}_{1}$ ADAPTIVE CONTROLLER

\begin{tabular}{|c|c|c|}
\hline Parameter & Description & Value \\
\hline$\Lambda$ & position error weight & $10 \times \operatorname{diag}(65,65,65,65)$ \\
\hline$A_{m}$ & transeint response matrix & $\operatorname{diag}(-6,-6,-6,-6)$ \\
\hline$\theta_{\max }$ & upper bound on $\theta$ & 50 \\
\hline$\sigma_{\max }$ & upper bound on $\sigma$ & 30 \\
\hline$\Gamma$ & adaptation gain & $10^{6}$ \\
\hline$K$ & noise rejection gain & $10^{3} \times \operatorname{diag}(6,6,6,6)$ \\
\hline$C(s)$ & $\mathcal{L}_{1}$ design filter & $144 /\left(s^{2}+21.6 s+144\right)$ \\
\hline
\end{tabular}



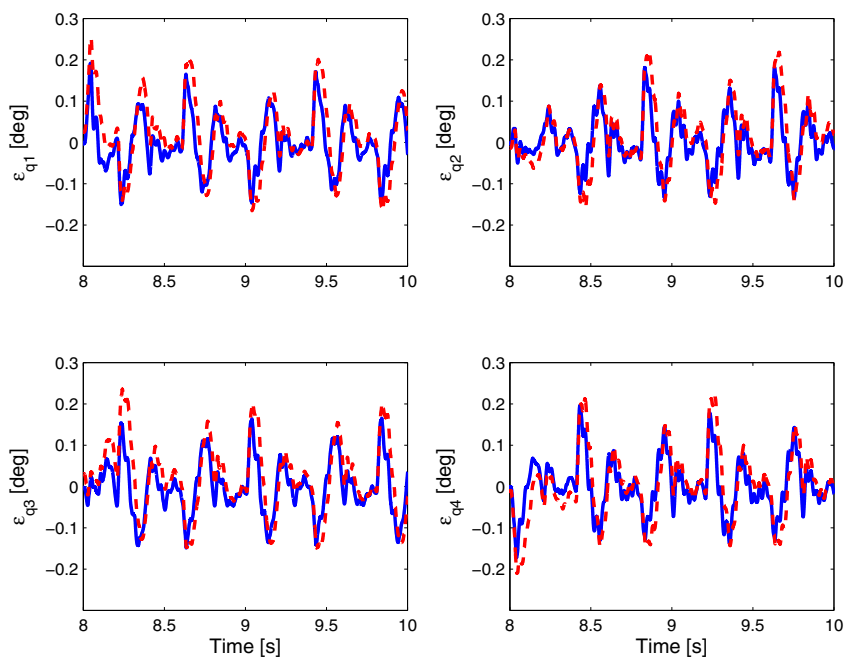

Fig. 4. Evolution of the joint tracking errors versus time: $\mathcal{L}_{1}$ adaptive controller (solid), PD (dashed)

rotational ones

$$
\begin{aligned}
\operatorname{RMS}_{q e r} & =\sqrt{\sum_{i=1}^{4} \operatorname{RMS}^{2}\left(\epsilon_{q i}\right)} \\
\operatorname{RMS}_{t r a} & =\sqrt{\operatorname{RMS}^{2}\left(\epsilon_{x}\right)+\operatorname{RMS}^{2}\left(\epsilon_{y}\right)+\operatorname{RMS}^{2}\left(\epsilon_{z}\right)} \\
\operatorname{RMS}_{r o t} & =\operatorname{RMS}\left(\epsilon_{\alpha}\right)
\end{aligned}
$$

In these formulas, $\epsilon_{(.)}$denotes the tracking error of the DOF (.). The obtained results are summarized in Table III, from which the improvement brought by the proposed controller is clearly observed. The tracking errors are reduced at least by $24 \%$ with respect of those of the PD controller. On the other hand, the improvement is more important for the rotation of the traveling plate with an improvement of at least $33 \%$.

For the $\mathcal{L}_{1}$ adaptive controller, the evolution of the estimated parameters $\hat{\theta}$ and $\hat{\sigma}$ is shown in Fig. 6 and Fig. 7 respectively. The estimated parameters keep oscillating and do not converge to a constant value since the desired trajectory of the traveling plate is time-varying. As expected, the estimated parameters never exceed their allowed bounds $\theta_{\max }$ and $\sigma_{\max }$ thanks to the projection operator. During the real-time experiments, the initial values $\hat{\theta}_{0}$ and $\hat{\sigma}_{0}$ were initialized to zero $\left(\hat{\theta}_{0}=[0,0,0,0]^{T}\right.$ and $\left.\hat{\sigma}_{0}=[0,0,0,0]^{T}\right)$. The generated control inputs are shown in Fig. 8. It can be noticed that the inputs are away from the limits of the actuators, namely $127 \mathrm{Nm}$. Moreover, they are smooth and

TABLE III

SCENARIO 1: TRACKING PERFORMANCE COMPARISON

\begin{tabular}{|c|c|c|c|}
\hline & $\mathrm{RMS}_{\text {qer }}[\mathrm{deg}]$ & $\mathrm{RMS}_{\text {tra }}[\mathrm{mm}]$ & $\mathrm{RMS}_{\text {rot }}[\mathrm{deg}]$ \\
\hline PD & 0.1402 & 0.57869 & 0.0138 \\
\hline $\mathcal{L}_{1}$ adaptive & 0.1028 & 0.44187 & 0.0093 \\
\hline Improvement & $27 \%$ & $24 \%$ & $33 \%$ \\
\hline
\end{tabular}
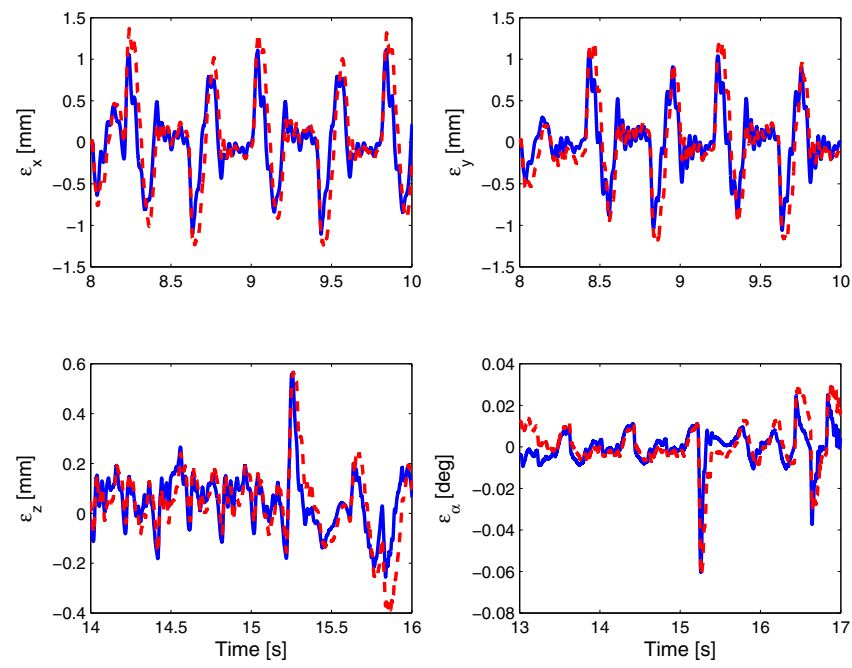

Fig. 5. Evolution of the Cartesian tracking errors versus time: $\mathcal{L}_{1}$ adaptive control (solid), PD (dashed)

they do not show show any discontinuities.

The movements of the robot while tracking reference trajectories have been recorded, they are illustrated in the video accompanying the paper.

\section{CONCLUSION AND FUTURE WORK}

This paper presents a first application of the recently developed $\mathcal{L}_{1}$ adaptive control theory to PKMs. The control law consists of two terms designed to track some desired reference trajectories. The first part being a stabilizing statefeedback term, while the second one is an adaptive term designed to compensate for the nonlinearities of the system. The proposed controller was implemented in real-time on a 4-DOFs PKM developed in our laboratory. To demonstrate its superiority, a comparison with a PD controller in terms of tracking performance was conducted. Experimental results show that the $\mathcal{L}_{1}$ adaptive controller gives better tracking performance, both in joint and task spaces. This work may be further extended by considering various operating conditions including payload changes, presence of external disturbances, etc. It would also be interesting to implement the proposed controller on other prototypes of PKMs.

\section{ACKNOWLEDGMENT}

This research was supported by the French National Research Agency (ANR) within the project ARROW.

\section{REFERENCES}

[1] J. P. Merlet, Parallel Robots, Second Edition. Dordrecht, Netherlands: Springer, 2006

[2] F. L.Lewis, D. M.Dawson, and C. T.Abdallah, Robot Manipulator Control, Theory and Practice. New York, USA: Marcel Dekker, Inc., 2004.

[3] P. R. Ouyang, W. Zhang, and F. Wu, "Nonlinear pd control for trajectory tracking with consideration of the design for control methodology," in Proc. IEEE International Conference on Robotics and Automation (ICRA'02), Washington, USA, May 2002, pp. 4126-4131.

[4] J. Luh, M. Walker, and R. Paul, "Resolved-acceleration control of mechanical manipulators," IEEE Trans. Automat. Contr., vol. 25, no. 3 , pp. 468-474, June 1980. 

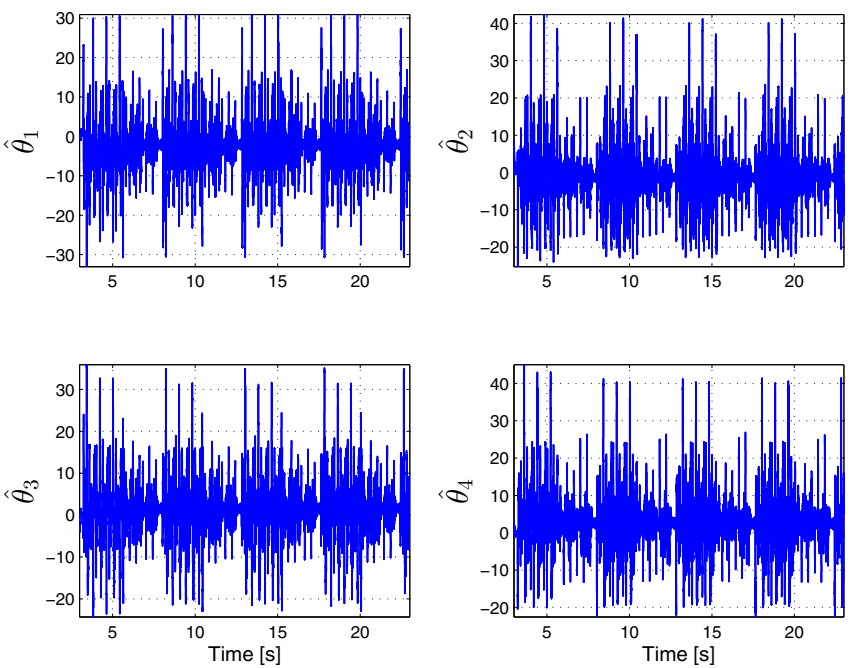

Fig. 6. Evolution of the estimated function $\hat{\theta}$ versus time
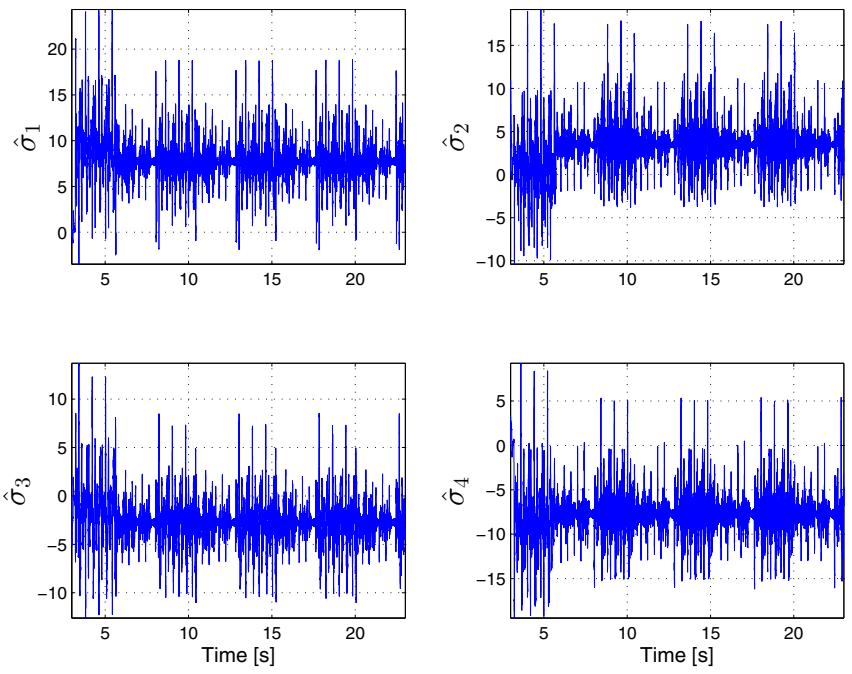

Fig. 7. Evolution of the estimated function $\hat{\sigma}$ versus time

[5] B. Paden and R. Panja, "Globally asymptotically stable pd+ controller for robot manipulators," International Journal of Control, vol. 47, no. 6, pp. 1697-1712, Jun 1988.

[6] D. Koditschek, "Natural motion for robot arms," in Proc. The 23rd IEEE Conference on Decision and Control (CDC'84), Las Vegas, Nevada, USA, Dec. 1984, pp. 733-735.

[7] S. Dubowsky and D. T. DesForges, "The application of modelreferenced adaptive control to robotic manipulators," Journal of Dynamic Systems, Measurement, and Control, vol. 101, no. 3, pp. 193 200, June 1979.

[8] J. J. Craig, P. Hsu, and S. S. Sastry, "Adaptive control of mechanical manipulators," The International Journal of Robotics Research, vol. 6, no. 2, pp. 16-28, June 1987.

[9] J.-J. E. Slotine, "On the adaptive control of robot manipulators," The International Journal of Robotics Research, vol. 6, no. 3, pp. 49-59, Sept. 1987.

[10] N. Sadegh and R. Horowitz, "Stability and robustness analysis of a class of adaptive controllers for robotic manipulators," The International Journal of Robotics Research, vol. 9, no. 3, pp. 74-92, June 1990.

[11] H. Cheng, Y.-K. Yiu, and Z. Li, "Dynamics and control of redundantly actuated parallel manipulators," IEEE/ASME Trans. Mechatron., vol. 8,
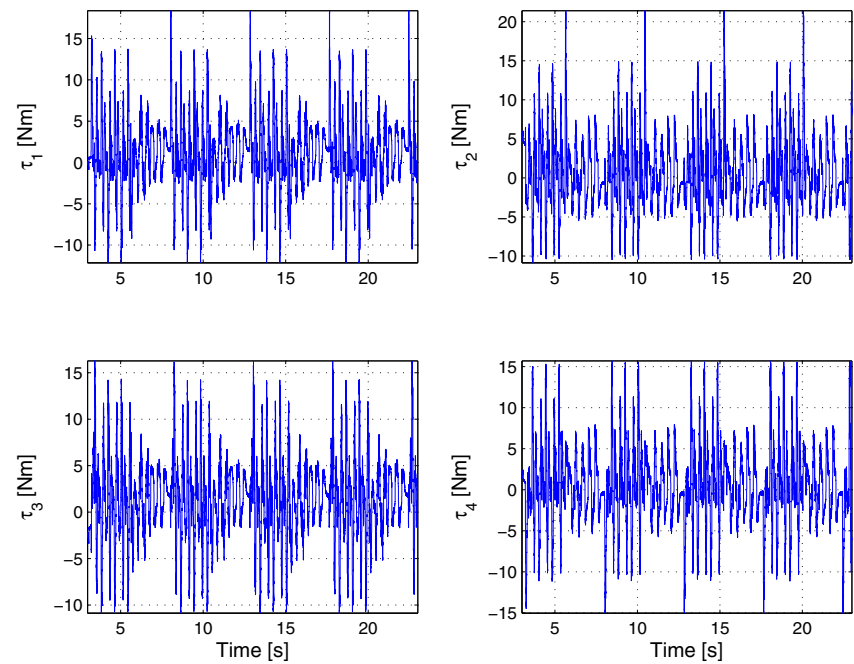

Fig. 8. Evolution of the adaptive $\mathcal{L}_{1}$ control inputs versus time

no. 4, pp. 483-491, Dec. 2003.

[12] M. Honegger, A. Codourey, and E. Burdet, "Adaptive control of the hexaglide, a 6 dof parallel manipulator," in Proc. IEEE International Conference on Robotics and Automation (ICRA'97), Albuquerque, New Mexico, USA, Apr. 1997, pp. 543-548.

[13] M. Bennehar, A. Chemori, and F. Pierrot, "A new extension of desired compensation adaptive control and its real-time application to redundantly actuated pkms," in Proc. IEEE International Conference on Intelligent Robots and Systems (IROS'14), Chicago, IL, USA, Sept. 2014, pp. 1670-1675.

[14] W. Shang and S. Cong, "Nonlinear adaptive task space control for a 2 dof redundantly actuated parallel manipulator," Nonlinear Dynamics, vol. 59, no. 1-2, pp. 61-72, Jan. 2010

[15] L. Wang, Z. Lu, X. Liu, K. Liu, and D. Zhang, "Adaptive control of a parallel robot via backstepping technique," The International Journal of Systems, Control and Communications, vol. 1, no. 3, pp. 312-324, Apr. 2009.

[16] C. Cao and N. Hovakimyan, "Design and analysis of a novel $\mathcal{L}_{1}$ adaptive controller, part i: Control signal and asymptotic stability," in American Control Conference (ACC'06), Minneapolis, USA, June 2006, pp. 3397-3402.

[17] — "Design and analysis of a novel $\mathcal{L}_{1}$ adaptive controller , part ii: Guaranteed transient performance," in American Control Conference (ACC'06), Minneapolis, USA, June 2006, pp. 3403-3408.

[18] N. Hovakimyan and C. Cao, L1 Adaptive Control Theory: Guaranteed Robustness with Fast Adaptation. Philadelphia, Pennsylvania, USA: SIAM, 2010.

[19] I. Gregory, C. Cao, E. Xargay, N. Hovakimyan, and X. Zou, "L1 adaptive control design for nasa airstar flight test vehicle," AIAA Guidance, Navigation, and Control Conference, p. 5738, Aug. 2009.

[20] M. Divine, C. Vincent, and C. Ahmed, "A novel application of multivariable $\mathcal{L}_{1}$ adaptive control: From design to real-time implementation on an underwater vehicle," in Proc. IEEE International Conference on Intelligent Robots and Systems (IROS'12), Vilamoura, Algarve, Portugal, Jan. 2012, pp. 76-81.

[21] K. D. Nguyen, H. Dankowicz, and N. Hovakimyan, "Marginal stability in $\mathcal{L}_{1}$ adaptive control of manipulators," in Proceedings of the 9th ASME International Conference on Multibody Systems, Nonlinear Dynamics, and Control, Portland, OR, USA, 2013.

[22] L. Techy, C. K. Reddy, C. A. Woolsey, C. Cao, and N. Hovakimyan, "Nonlinear control of a novel two-link pendulum," in American Control Conference (ACC'07), New York, USA, 2007, pp. 19-24.

[23] D. Corbel, M. Gouttefarde, and O. Company, "Towards 100g with $\mathrm{pkm}$. is actuation redundancy a good solution for pick and place?" in Proc. IEEE International Conference on Robotics and Automation (ICRA'10), Anchorage, Alaska, May 2010, pp. 4675-4682. 